\title{
Sanofi-Cell Research outstanding paper award of 2016
}

Cell Research (2017) 27:1299. doi:10.1038/cr.2017.137; published online 3 November 2017

We are pleased to announce winners of the 2016 Sanofi-Cell Research Outstanding Research Article Award: Drs Ya-Ping Zhang and Peter Savolainen, for their paper entitled "Out of southern East Asia: the natural history of domestic dogs across the world"; Drs Zhen-Ge Luo and Cheng-Feng Qin, for their paper entitled "Vertical transmission of Zika virus targeting the radial glial cells affects cortex development of offspring mice". The 2016 Sanofi-Cell Research Outstanding Review Article Award goes to Dr David Komander, for his review paper entitled "Ubiquitin modifications". The award consists of a prize of $€ 3000$ for the Outstanding Review Article Award and $€ 5000$ for the Outstanding Research Article Award sponsored by Sanofi.

The origin and evolution of the domestic dog remains controversial. Here, in the January issue of 2016, Drs Ya-Ping Zhang, Peter Savolainen and their colleagues [1] analyzed the whole genome sequences from a total of 58 canids from across the world, which revealed that dogs from southern East Asia have significantly higher genetic diversity compared to other populations. This study, for the first time, indicated an ancient origin of domestic dogs in southern East Asia 33000 years ago.
Dr Ya-Ping Zhang

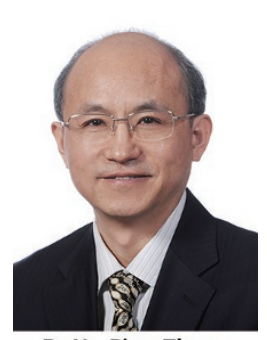

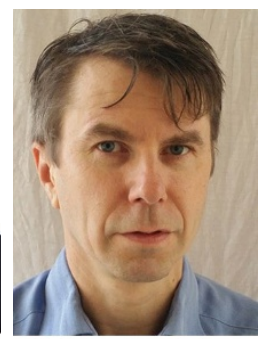

Dr Peter Savolainen
The outbreak of Zika virus (ZIKV) infection in Latin America associated with microcephaly in newborns was declared as a Public Health Emergency of International Concern in February 2016. In the June issue last year, Drs ZhenGe Luo and Cheng-Feng Qin and their colleagues [2] established the causal link between maternal ZIKV infection and malformation of the fetal brain in a mouse model. This study thus provides a useful animal model for the evaluation of therapeutic approaches or preventive strategies against ZIKV infections. The award-winning review by Dr David Ko-

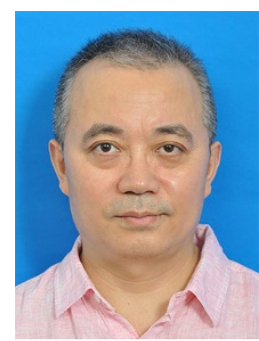

Dr Zhen-Ge Luo

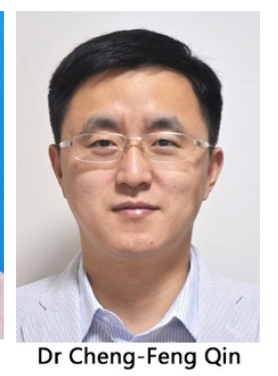

mander [3] was published in the April issue of 2016, as part of a special issue on ubiquitin and ubiquitin-like protein (Ubl) signaling [4] featuring a collection of seven authoritative reviews from worldwide renowned experts in the field. This review presents an overview of several types of ubiquitin-protein modifications in cells and the distinct roles of specific polyUb chains in cellular signaling. It also summarizes the recent findings in the field of ubiquitin acetylation and phosphorylation.

Please join us to congratulate Drs Zhang and Savolainen, Luo and Qin, and Komander on their winning of the 2016 Sanofi-Cell Research Outstanding

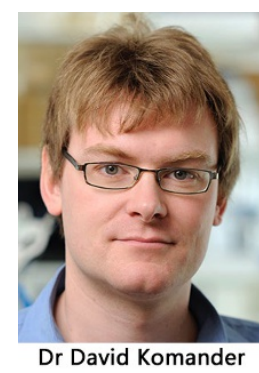

Paper Award.

Editorial Office ${ }^{1}$

${ }^{1}$ Cell Research, Shanghai Institutes for Biological Sciences, Chinese Academy of Sciences, Shanghai 200031, China

cellres@sibs.ac.cn

\section{References}

1 Wang GD, Zhai W, Yang HC, et al. Out of southern East Asia: the natural history of domestic dogs across the world. Cell Res 2016; 26:21-33.

2 Wu KY, Zuo GL, Li XF, et al. Vertical transmission of Zika virus targeting the radial glial cells affects cortex development of offspring mice. Cell Res 2016; 26:645-654.

3 Swatek KN, Komander D. Ubiquitin modifications. Cell Res 2016; 26:399-422.

$4 \mathrm{Hu}$ R, Hochstrasser M. Recent progress in ubiquitin and ubiquitin-like protein (Ubl) signaling. Cell Res 2016; 26:389-390. 